\title{
Detection of Texture and Isolated Features Using Alternating Morphological Filters
}

\author{
Igor Zingman ${ }^{1}$, Dietmar Saupe ${ }^{1}$, and Karsten Lambers ${ }^{2}$ \\ 1 Department of Computer and Information Science, \\ University of Konstanz, Germany \\ 2 Institute of Archaeology, Heritage Sciences and Art History, \\ University of Bamberg, Germany
}

\begin{abstract}
Recently, we introduced a morphological texture contrast (MTC) operator that allows detection of textural and non-texture regions in images. In this paper we provide comparison of the MTC with other available techniques. We show that, in contrast to other approaches, the MTC discriminates between texture details and isolated features, and does not extend borders of texture regions. Using the ideas underlying the MTC operator, we develop a complementary operator called morphological feature contrast (MFC) that allows extraction of isolated features while not being confused by texture details. We illustrate an application of the MFC operator for extraction of isolated objects such as individual trees or buildings that should be distinguished from forests or urban centers. We furthermore provide an example of how this operator can be used for detection of isolated linear structures. We also derive an extended version of the MFC that works with vector-valued images.
\end{abstract}

\section{Introduction}

This paper focuses on the problem of distinguishing isolated features, also called individual features, from features that are part of a texture 1 . We refer to the latter features as texture details. This problem may occur when one wants to detect texture regions, but distinguish them from isolated features that should not be assigned to a texture class. The dual problem occurs when it is necessary to detect isolated features avoiding detection of parts of neighboring or background texture even if texture details are similar to features of interest. Here we consider both problems, namely detection of texture and of individual features.

Although a large variety of texture classification methods has been developed, much less attention has been given to the apparently simpler problem of texture detection that discriminates between high contrast texture (of any type) and non-texture regions. This is not a simple task if accurate localization is required and if texture must be distinguished from individual features.

In [1] it was proposed to use the difference between maximal and minimal intensities (MaxMin difference) in a pixel neighborhood for a fast segmentation

${ }^{1}$ By features we mean small image elements, for example blobs, ridges or edges. 
of an image into textured and non-texture regions. A standard deviation (StD) is frequently used as a measure of texture that describes its smoothness [2]. In [3], where the Local Binary Patterns (LBP) approach was developed, the authors also suggested to incorporate a variance-based descriptor for texture classification purposes. While the LBP descriptor is related to inherent texture properties, a complementary variance-based descriptor measures texture contrast. The amplitude modulation function (AMF), derived from the amplitude-modulation frequency-modulation model [4], can locally capture texture contrast. Though each of the texture contrast descriptors mentioned above can be used to discriminate between texture regions and non-texture areas, also called smooth areas in this paper, they cannot distinguish individual features from texture details.

Recently, several descriptors were suggested to approach this problem. In [5] the PanTex index was developed to detect settlements in panchromatic satellite imagery. The operator is able to distinguish texture areas from individual linear features such as roads or borders between homogenous cultivated fields in satellite images. The PanTex index is defined as a minimal contrast among contrast measures derived from the gray-level co-occurrence matrixes (GLCM) [6], computed for different orientations of displacement vectors. The PanTex method, however, does not distinguish other individual features, such as isolated peaks or small isotropic blobs, from texture.

The component count (CC) method [7] is based on the product of two measures computed in small image blocks. The first one is the sum of the number of connected components (component count) in the background and the foreground obtained by simple binarization of image blocks. The second measure is the difference between average intensities in the background and the foreground. This descriptor is supposed to discriminate blocks covering texture and individual step edges at the borders between homogenous regions. A similar idea of counting the number of local extrema (texture primitives) for detection of texture regions was proposed earlier in [8]. Since this method does not take into account contrast of texture primitives, it can be very sensitive to noise.

Another disadvantage that all the above texture descriptors have in common, is that they extend or blur the edges of texture regions preventing accurate localization of texture borders. Recently, we introduced a morphological texture contrast (MTC) descriptor that does not suffer from the above disadvantages [9]. This operator, briefly reviewed in Sec. 2, measures the difference between upper and lower texture envelopes estimated by means of alternating morphological filters [10. Its qualitative performance was illustrated in [9] using only few remotely sensed images and no quantitative comparison was provided. In Sec. 3 we provide a quantitative comparison using artificially created images and qualitative comparison using a set of standard test images.

As we stated in the beginning of this section, the dual problem to the problem of texture detection is detection of individual features while distinguishing them from texture details. This problem has mainly been treated in the context of edge detection capable of discarding texture surroundings. For example, recently in [1] a surround inhibition mechanism was introduced to improve edge detection 
at region boundaries. In 12 a normal complexity measure was proposed that is able to separate isolated curves and isolated edges from texture in binary images. The paper provides an original theoretical framework, but it seems to be computationally very expensive.

In Sec. 4 we show how the ideas underlying the MTC operator lead to a Morphological Feature Contrast (MFC) operator that aims at detection of small isolated objects, rather than edges, in textured background. We illustrate the potential of the MFC operator on gray-scale images and also derive its extension to vector-valued images. Additionally, we show how the MFC operator can be incorporated into a scheme for extraction of isolated linear structures.

\section{Detection of Texture Regions: The Morphological Texture Contrast (MTC) Operator}

Below, we define the morphological texture contrast (MTC) transformation $\psi_{\text {MTC }}(f)$ that we recently introduced in 9] for localization of high contrast textured regions. It uses alternating morphological filters, $\gamma_{r} \varphi_{r}$ and $\varphi_{r} \gamma_{r}$, which are closing $\varphi$ followed by opening $\gamma$ and opening followed by closing, respectively. $r$ denotes the size of the structuring element (SE). Alternating filters are usually employed for noise filtering. We use them to estimate texture envelopes. The difference between upper and lower envelope 22 defines a measure of texture contrast, which can serve as an indicator of the presence of texture

$$
\psi_{\mathrm{MTC}}(f)=\left|\gamma_{r} \varphi_{r}(f)-\varphi_{r} \gamma_{r}(f)\right|^{+},
$$

where the argument $f$ denotes a $1 \mathrm{D}$ signal or a 2D gray-scale image, and $|\cdot|^{+}$ is defined as

$$
|\nu|^{+} \triangleq \begin{cases}\nu, & \nu>0 \\ 0, & \text { otherwise } .\end{cases}
$$

A remarkable property of these envelopes is that they coincide at individual features, thereby yielding low response at individual features even if they are of high contrast.

The results of applying this transformation to an artificial 1D signal and to a remotely sensed image of a forested area are shown in Fig. 11 and Fig. 2(a,b), respectively. Throughout this paper we use a square SE, where the size refers to its side length. The size $r$ of the SE in Eq. (1) should be chosen to be larger than the maximal distance between details in textured regions. Features that stand apart from texture details farther than $r$ are treated as individual features and are suppressed correspondingly. In general, we can use different sizes $r_{1} \neq r_{2}$ for SEs in $\left|\gamma_{r_{2}} \varphi_{r_{1}}(f)-\varphi_{r_{2}} \gamma_{r_{1}}(f)\right|^{+}$. While $r_{1}$ should be chosen to be larger than the maximal distance between texture details, $r_{2}$ should be chosen to be smaller

\footnotetext{
${ }^{2}$ Since in the 2D case, $\varphi_{r} \gamma_{r}$ and $\gamma_{r} \varphi_{r}$ are not ordered [13, a lower envelope might be above an upper envelope. However, it can be shown that regions where this happens are small in the sense that an erosion with a structuring element of size $r$ completely removes these regions.
} 

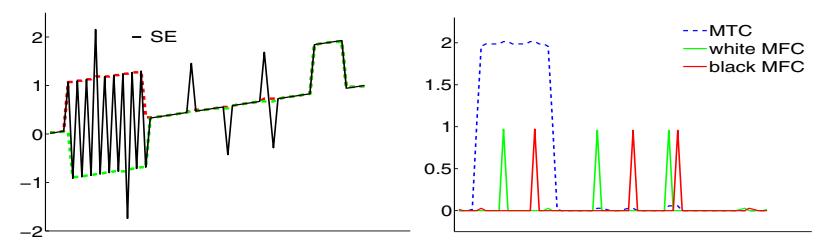

Fig. 1. Left: An artificial signal composed of a slow varying component, a texture region, and individual features. Upper and lower envelopes of the texture obtained with alternating morphological filters are shown by red and green dashed lines. Right: Extraction of the texture region and individual features with the MTC (Sec. 2) and the MFC (Sec. 4) operators.

than the minimal size of texture regions. Thus, using $r_{1} \neq r_{2}$ adds an additional degree of control of the minimal number of clustered details to be regarded as a texture. Comparison of Fig. 2(b) and (c) illustrates this point.

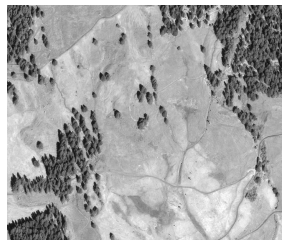

(a)

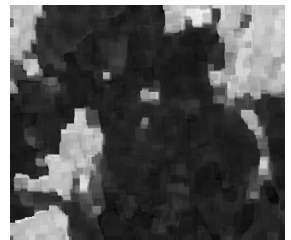

(b)

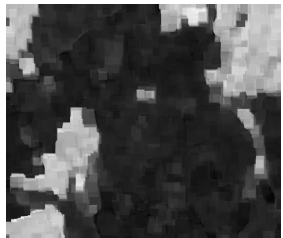

(c)

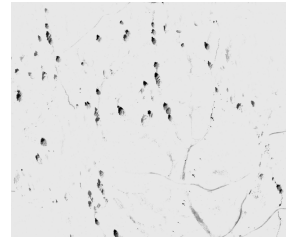

(d)

Fig. 2. (a) Pan-chromatic satellite image of $1360 \times 1160$ pixel size ${ }^{4}$. (b) and (c) The MTC descriptor. $r_{1}=r_{2}=30$ in (b) and $r_{1}=30, r_{2}=35$ in (c). (d) Extraction of individual dark features, i.e. individual trees, using the $\psi_{\mathrm{MFC}}^{-}$operator $^{5}$ with $r_{1}=r_{2}=30$ (Sec. (4). Note that trees in forest areas are almost completely suppressed.

The MTC operator is bias invariant, $\psi_{\mathrm{MTC}}(f)=\psi_{\mathrm{MTC}}(f+a)$, where $a \in \mathbb{R}$ is a constant, invariant to signal inversion $3, \psi_{\mathrm{MTC}}(f)=\psi_{\mathrm{MTC}}(a-f)$, and proportional to signal magnitude $\psi_{\mathrm{MTC}}(a f)=a \psi_{\mathrm{MTC}}(f)$. An important property of the MTC transformation is that it neither extends nor blurs the edges of textured regions, thereby allowing accurate localization of texture borders. This property is illustrated in Fig. 3 in the rightmost column.

\section{Comparison of Texture Contrast Descriptors}

In this section we qualitatively and quantitatively compare the performance of the MTC algorithm with the CC, the MaxMin difference, the StD, the LBP

\footnotetext{
${ }^{3}$ Bias invariant and self-complementary operators 14 are invariant to signal inversion.

4 The image was captured by the GeoEye-1 satellite (C) GeoEye 2011).

${ }^{5}$ High values of the transformation are represented by dark tones.
} 
contrast, the AMF, and the PanTex algorithms. We denote by $w$ a scale parameter required for all algorithms. To allow a consistent comparison, a few algorithms were slightly modified as follows.

In the $\mathrm{CC}$ algorithm we avoided several parameters suggested by the authors since they need to be adjusted for each type of image. Specifically, we used the simple product of contrast and number of connected components. Instead of disjoint blocks, a sliding window of the single size $w$ was used to compute the texture measure at each pixel as in the other compared methods. In the PanTex algorithm we used a square root of contrast measure derived from the GLCM matrix. This contrast measure can actually be computed without explicit calculation of the GLCM matrix. The GLCM contrast measure was estimated within a window of arbitrary size $w$, such that displacement vectors of the length $\frac{w}{3}$ were determined by all pixels on a circle of radius $\frac{w}{3}$.

To compute the LBP local variance and the AMF measures we used a Matlab code available online 6 . In the LBP we used the square root of local variance computed as a variance of $4(w-1)$ equally spaced point samples on a circle of radius $\frac{w}{2}$. In the $\mathrm{AMF}$ approach we set the largest period of a sinusoid in the Gabor filters to $3 w$ pixels. Note that similar to the MTC operator, all the algorithms, after the small modifications described above (except for AMF), are bias invariant, invariant to signal inversion and proportional to signal magnitude.

\subsection{Qualitative Comparison}

The contrast descriptors obtained using the compared transformations are shown in the first three columns in Fig. 3. All the descriptors have high values in textured areas and low values in smooth areas. However, contrary to the MTC operator, the other approaches yield also high responses at isolated features (edges, ridges, peaks) that do not belong to texture. The PanTex descriptor partially succeeded to suppress isolated curvilinear structures.

To better visualize the accuracy in texture localization, the texture descriptors were superimposed on the enlarged part of the satellite image in the forth column of Fig. 3. where the contrast of red tones is proportional to the values of the descriptors. Since the distribution of descriptor values is strongly bimodal, one can distinguish two major levels of texture descriptors, low and high, that appear as a gray-reddish and saturated red overlayed on the original image. As can be seen from these images, another advantage of the MTC operator is that it does not extend the borders of texture regions as other methods do. The CC method does not extend borders of texture regions, instead it generates a halo near texture borders and around individual features. This effect does not occur in the original version of the $\mathrm{CC}$ method, in which disjoint/overlapping block processing was performed (which, however, would not allow accurate texture localization). The LBP method also leaves rings around individual trees due to high response at distances equal to the radius of the circle used to compute the variance of samples on it.

\footnotetext{
${ }^{6}$ Matlab sources are available at http://www.cse.oulu.fi/CMV/Downloads/LBPMatlab and http://cvsp.cs.ntua.gr/software/texture/
} 


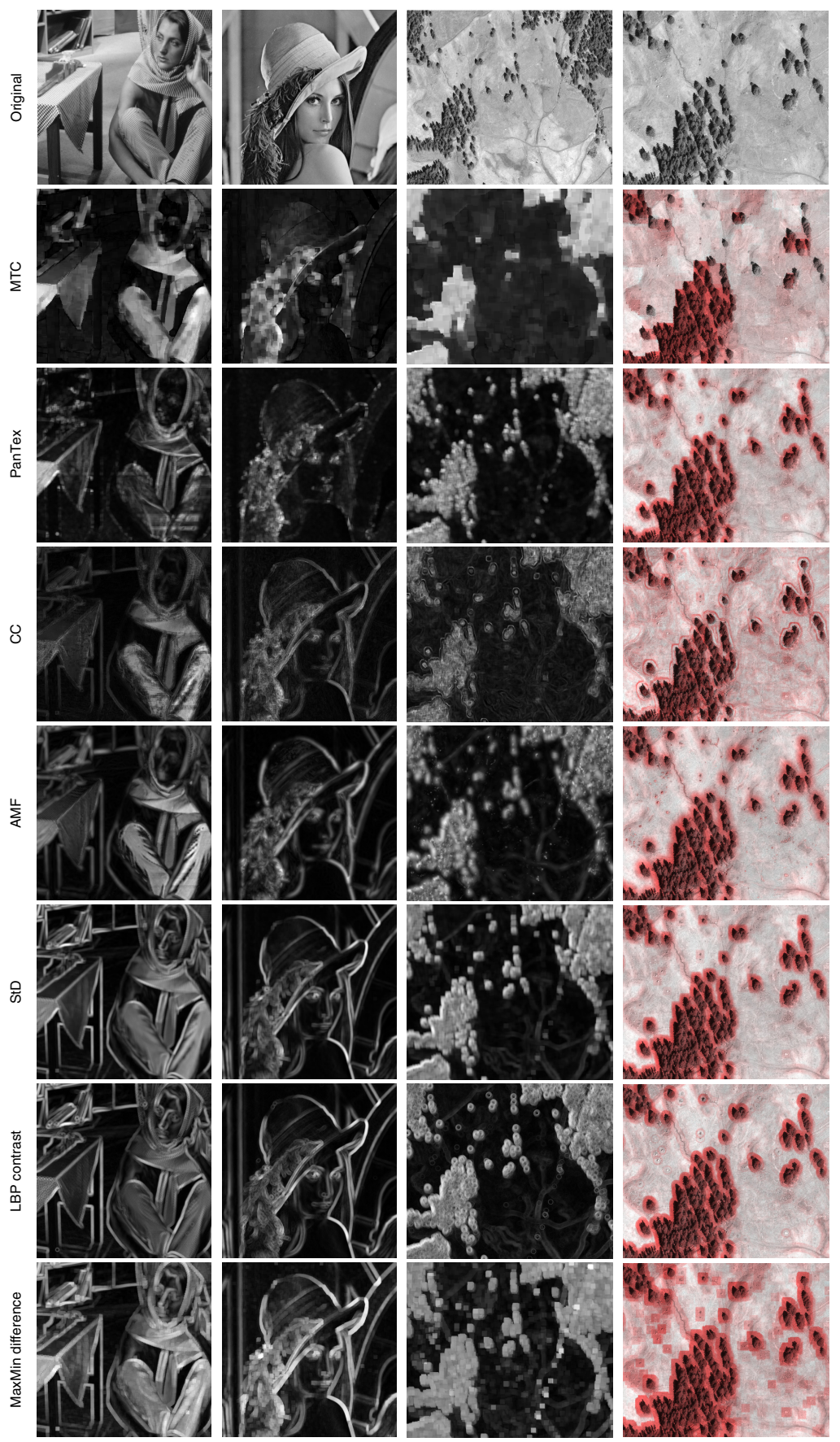

Fig. 3. Comparison of texture contrast descriptors. The first two original images are of size 512x512 pixels; the third image is the same as in Fig. 2(a); the forth image is an enlarged part of the third image. $w=10$ for the first two images and $w=30$ for the satellite images. Further details can be found in Sec. 3 and Sec. 3.1. 


\subsection{Quantitative Comparison}

In order to quantitatively compare the ability of the methods to distinguish between texture and non-texture areas we quantify separability between distributions of texture descriptors in these areas. We used the Fisher criterion [15] that measures the distance between distribution means relative to their compactness. The criterion is given by $\frac{\left(\mu_{1}-\mu_{2}\right)^{2}}{\sigma_{1}^{2}+\sigma_{2}^{2}}$, where $\mu$ denotes a class mean and $\sigma^{2}$ denotes a class variance. Since ground truth data is required to define textured and nontexture regions, we created an artificial data set of gray-scale images along with corresponding masks of texture regions and non-texture areas, whereby the last also include individual features.

The data set consists of 100 images of 300x300 pixels with circular texture clusters and individual features (clusters may overlap, see the upper-left image in Fig. (5). The number of clusters and their diameters were chosen uniformly randomly and varied from 2 to 4 and from 60 to 120 pixels, respectively. The diameter of both individual features and texture details was 5 pixels. Texture details within clusters were placed at positions on a regular grid with random Gaussian offsets. The distance between nodes of the regular grid was set to 9 pixels. The amplitude of texture details and individual features varied randomly with normal distribution. White noise was added followed by smoothing with an averaging filter with a $3 \times 3$ kernel. The standard deviation of the noise was equal to one third of the amplitude of the texture details.

In the first two experiments we set the mean amplitude of individual features equal and triple, respectively, of the amplitude mean of texture details. Fig. 4(a, b) show the resulting separability measure for the size parameter $w$ from 10 to 70 pixels. The figures show superiority of the MTC operator when discriminating non-texture regions from texture areas. These figures also reveal a high degree of immunity of the MTC approach to individual features with high magnitude.

In the third experiment we restrict the class of non-texture areas to areas adjacent to texture regions and to individual features including their neighborhood. Fig. 4(c) shows separability between such restricted non-texture areas and texture regions when mean amplitude of texture details and individual features were equal. A comparison of Fig. 4(c) and Fig. 4(a) confirms that the superiority in the performance of the MTC operator stems from its ability to distinguish texture from isolated features as well as from regions adjacent to texture borders.

\section{Extraction of Isolated Features: The Morphological Feature Contrast (MFC) Operator}

Using the ideas underlying the MTC operator, below we propose a Morphological Feature Contrast (MFC) operator that extracts isolated structures while suppressing texture details of textured background. Using alternating morphological filters, upper and lower texture envelopes were estimated in the MTC approach. To extract bright or dark individual features, we suggest using the difference between the original signal and one of its envelopes, as defined in the 


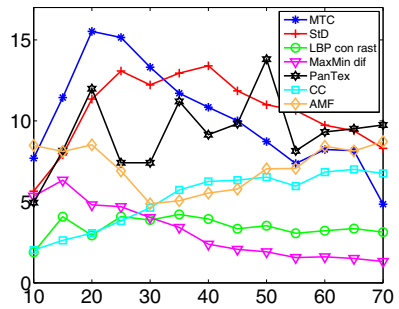

(a)

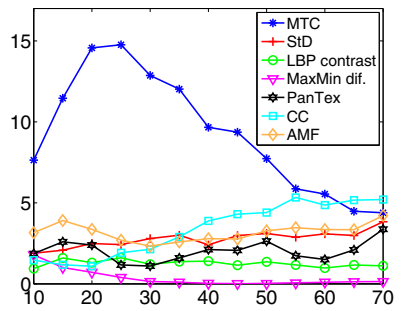

(b)

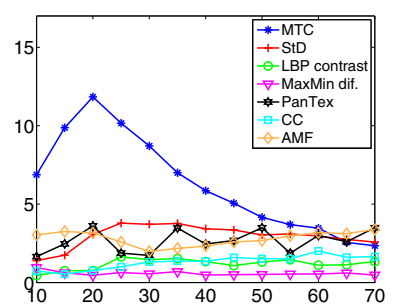

(c)

Fig. 4. (a) and (b): The measure of separability between texture and non-texture regions as a function of the scale parameter $w$. The mean amplitude of individual features is equal to the amplitude of texture details in (a) and tripled in (b). (c) The measure of separability of texture regions from areas around individual features and smooth areas adjacent to texture borders. The mean amplitude of individual features equals the amplitude of texture details.

following equations

$$
\begin{aligned}
& \psi_{\mathrm{MFC}}^{+}(f)=\left|f-\gamma_{r_{2}} \varphi_{r_{1}}(f)\right|^{+}, \\
& \psi_{\mathrm{MFC}}^{-}(f)=\left|\varphi_{r_{2}} \gamma_{r_{1}}(f)-f\right|^{+} .
\end{aligned}
$$

If one is interested in both dark and bright features, $\psi_{\mathrm{MFC}}=\psi_{\mathrm{MFC}}^{+}+\psi_{\mathrm{MFC}}^{-}$ should be used. We call $\psi_{\mathrm{MFC}}^{+}$and $\psi_{\mathrm{MFC}}^{-}$white and black MFC, respectively. Similar to the MTC transformation, $\psi_{\mathrm{MFC}}$ is bias invariant, invariant to signal inversion, and proportional to signal magnitude. The MFC operators applied to a 1D artificial signal are illustrated in Fig. 11 The size $r_{1}$ of the SE should be greater than the maximal distance between details of texture to be suppressed. The size $r_{2}$ should be greater than the size of features to be extracted. If not stated otherwise, we use equal sizes for both SEs.

It can be shown that $\psi_{\mathrm{MFC}}$ is equal to $\max (\varphi \gamma(f), f)-\min (\gamma \varphi(f), f)$, while $\psi_{\mathrm{MFC}}^{+}$and $\psi_{\mathrm{MFC}}^{-}$are equal to operators that were already defined in [16] as $f-\min (\gamma \varphi(f), f)$ and $\max (\varphi \gamma(f), f)-f$, and were used for detection of defects in the noisy background of a metallic surface.

Fig. 5 and Fig. 2(d) show examples of the MFC operators $\psi_{\mathrm{MFC}}$ and $\psi_{\mathrm{MFC}}^{-}$ applied to gray-scale images. One can observe that various individual features/ objects were highlighted while texture areas were simultaneously suppressed. For example, in the middle image in Fig. 5 the forest texture area and the texture of the village center on the right were suppressed, while isolated buildings outside the dense village center were preserved in the output image.

The MFC operator is capable of suppressing texture areas even if composed of details of higher magnitude and similar shape in relation with the magnitude and shape of individual features. Although several methods were developed to extract object boundaries (edge features) from textured background, we are not aware of other techniques that perform qualitatively similar to the MFC when extracting blob-like features (as well as features of an arbitrary shape). 

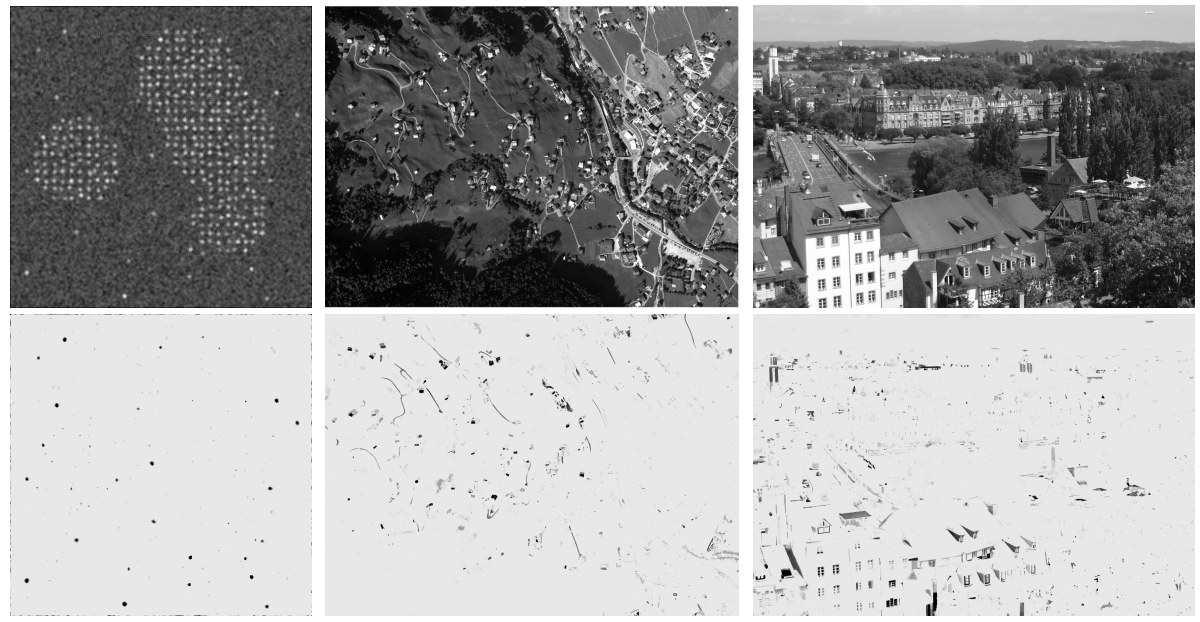

Fig. 5. First row: $312 \times 312$ artificial, 2570x1870 satellite $^{4}$, and $1010 \times 690$ digital camera images. Second row: individual features extracted by means of the MFC operator $\psi_{\mathrm{MFC}}{ }^{5} \cdot r_{1}=30, r_{2}=10$ for the artificial image; $r_{1}=r_{2}=90$ for the satellite image, and $r_{1}=r_{2}=25$ pixels for the digital camera image.

\subsection{Application of the MFC Operator to Extraction of Isolated Linear Features}

Above we have shown that the MFC operators are capable of extracting features of different types with width smaller than $r_{2}$. Specific features can be extracted by a sequence of standard morphological transformations, with the structuring element shaped similarly to features. Here we illustrate advantages of the use of the MFC within such a sequence for the case of linear features.

The remotely sensed images in Fig. 6] (left) contain rectangular structures composed of linear walls, which used to be livestock enclosures. A black tophat transform removes background and emphasizes small dark structures in the image. A subsequent filter $\gamma_{\text {lin }}$ obtained by the point-wise maximum of morphological openings with linear SE at different orientations extracts narrow linear structures longer than the length of the structuring element. In the resulting image shown in Fig. [6 (middle) linear walls were highlighted but also texture details were emphasized. Furthermore, an appropriate threshold setting is required to obtain a binary map of features.

To remove texture while keeping isolated features, the MFC operator $\psi_{\mathrm{MFC}}^{+}$ may be applied 7 prior to $\gamma_{\text {lin }}$. This sequence of operators completely removes most texture details. Non-zero pixels of the resulting image are shown in black in Fig. [6 (right). No threshold selection was required to obtain the binary map of linear features. We are currently evaluating the potential of using the

7 The $\psi_{\mathrm{MFC}}^{-}$could also be directly applied to the initial image. 

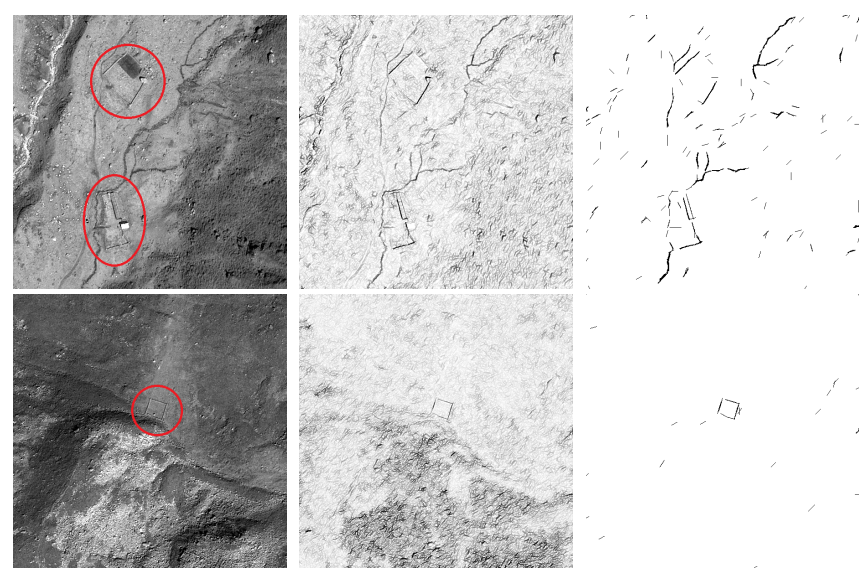

Fig. 6. Left: Satellite images ${ }^{4}$ of $600 \times 600$ pixels size with man-made structures (linear walls). Middle: Black top-hat transform followed by an opening filter $\gamma_{\text {lin }}$ obtained by the point-wise maximum of morphological openings with linear structuring elements at different orientations ${ }^{5}$. Right: Black top-hat transform followed by $\psi_{\mathrm{MFC}}^{+}$and subsequent $\gamma_{\text {lin }}$. Non-zero pixels are shown in black. Parameters: 5x5 SE in the top-hat operator, $r_{1}=5, r_{2}=10$ in $\psi_{\mathrm{MFC}}^{+}$; the length of the linear SE in $\gamma_{\text {lin }}$ equals 15 pixels.

MFC operators for extraction of linear features within the Silvretta Historica project8.

\subsection{Extension of the MFC Operator to Vector-Valued Images}

In this section we generalize the MFC operators from gray-scale to vector-valued discrete images, where each pixel is attributed by a vector of values. A multispectral image is an example of such a vector-valued image. The problem with extending morphological operators to vector-valued images lies in the lack of a natural ordering of vectors. However, some morphological transformations defined in terms of arithmetic differences between morphological operators can naturally be extended to vector-valued images without the need to chose a vectorial order. Examples of such extended transformations were recently proposed for morphological gradient and for top-hat in [18, [19]. Using similar ideas we derive an extended version of the MFC operators below.

The MFC operator defined in Eq. (3) can be rewritten in the following form $\psi_{\mathrm{MFC}}^{-}(f)=\left|\varepsilon_{r_{2}} \delta_{r_{2}} \delta_{r_{1}} \varepsilon_{r_{1}}(f)-f\right|^{+}$where $\delta$ and $\varepsilon$ denote morphological dilation and erosion, respectively. $r_{1}, r_{2}$ denote sizes of SEs $B_{p}^{(1)}$ and $B_{p}^{(2)}$, respectively, centered at $p$. Omitting the details of derivation, the last equation can further be transformed to

$$
\left[\psi_{\mathrm{MFC}}^{-}(f)\right](x)=\min _{k \in B_{x}^{(2)}} \max _{j \in B_{k}^{(3)}} \min _{i \in B_{j}^{(1)}}|f(i)-f(x)|^{+},
$$

\footnotetext{
${ }^{8}$ Details on this project can be found in 17 .
} 
where $B^{(3)}$ denotes the structuring element $B^{(1)}$ dilated by $B^{(2)}$. Similarly,

$$
\left[\psi_{\mathrm{MFC}}^{+}(f)\right](x)=\min _{k \in B_{x}^{(2)}} \max _{j \in B_{k}^{(3)}} \min _{i \in B_{j}^{(1)}}|f(x)-f(i)|^{+} .
$$

We now define a new vectorial MFC operator $\psi_{\mathrm{MFC}}(\bar{f})$ that applies to vectorvalued images $\bar{f}$. We replace the non-negative difference between intensity values in the last two equations by a suitable metric distance $D$ between vectors,

$$
\left[\psi_{\mathrm{MFC}}(\bar{f})\right](x)=\min _{k \in B_{x}^{(2)}} \max _{j \in B_{k}^{(3)}} \min _{i \in B_{j}^{(1)}} D(\bar{f}(x), \bar{f}(i)) .
$$

In contrast to $\psi_{\mathrm{MFC}}^{+}(f)$ and $\psi_{\mathrm{MFC}}^{-}(f)$, the $\psi_{\mathrm{MFC}}(\bar{f})$ operator extracts both dark and bright structures when applied to multispectral images. If one is interested in extraction of either dark or bright features only, pseudo-distances may be used. For example, instead of using $D_{\infty}$ distance, pseudo distances defined by $D_{\infty}^{+}(\bar{f}, \bar{g})=\max _{i}\left|f_{i}-g_{i}\right|^{+}$and $D_{\infty}^{-}(\bar{f}, \bar{g})=\max _{i}\left|g_{i}-f_{i}\right|^{+}$may be employed.

Vectorial operators may be preferable to independent processing of channels of a vector-valued image followed by integration of the results. On the other hand, in many cases, independent processing produces similar or even better results due to smaller levels of output noise. A comparative evaluation of the vectorial MFCs is beyond the scope of this paper.

\section{Summary}

We have shown how alternating morphological filters can be used to design operators for detection of texture regions and isolated features. The comparison of the morphological texture contrast (MTC) operator based on visual inspection and quantitative experiments, reveals its superiority over other methods used for texture detection. This operator is very attractive for various applications due to its ability to discriminate texture from isolated features irrespectively of their high magnitude, good localization properties, and simplicity. The complementary morphological feature contrast (MFC) operator was proposed for extraction of isolated features in images containing also texture background. We show that the MTC and the MFC have a similar structure and are of special interest where it is important to distinguish isolated features from texture details. An extension of the MFC operator was derived that allows the MFC operator to work directly with vector-valued images. We have also proposed a simple scheme based on the MFC operator for detection of isolated linear structures.

Acknowledgments. This work was funded by the Interreg IV Program "Alpenrhein - Bodensee - Hochrhein" and by the Zukunftskolleg, University of Konstanz. It was also supported by the DFG Research Training Group GK-1042 "Explorative Analysis and Visualization of Large Information Spaces." 


\section{References}

1. Dinstein, I., Fong, A., Ni, L., Wong, K.: Fast discrimination between homogeneous and textured regions. In: Proc. of Int. Conf. on Pattern Recognition, Montreal, Canada (1984)

2. Gonzalez, R.C., Woods, R.E.: Digital Image Processing (2001)

3. Ojala, T., Pietikäinen, M., Mäenpää, T.: Multiresolution gray-scale and rotation invariant texture classification with local binary patterns. IEEE Trans. Pattern Anal. Mach. Intell. 24, 971-987 (2002)

4. Kokkinos, I., Evangelopoulos, G., Maragos, P.: Texture analysis and segmentation using modulation features, generative models and weighted curve evolution. IEEE Trans. Pattern Analyis and Machine Intelligence 31, 142-157 (2009)

5. Pesaresi, M., Gerhardinger, A., Kayitakire, F.: A robust built-up area presence index by anisitropic rotation-invariant textural measure. IEEE Journal of Selected Topics in Applied Earth Observation an Remote Sensing 1 (September 2008)

6. Haralick, R.M., Shanmugam, K., Dinstein, I.: Textural features for image classification. IEEE Trans. Systems Man and Cybernetics 6, 610-621 (1973)

7. Bergman, R., Nachlieli, H., Ruckenstein, G.: Detection of textured areas in natural images using an indicator based on component counts. J. Electronic Imaging 17(4) (2008)

8. Karu, K., Jain, A.K., Bolle, R.M.: Is there any texture in the image? Pattern Recognition 29, 1437-1446 (1996)

9. Zingman, I., Saupe, D., Lambers, K.: Morphological operators for segmentation of high contrast textured regions in remotely sensed imagery. In: Proc. of the IEEE Int. Geoscience and Remote Sensing Symposium, Munich, Germany (July 2012)

10. Serra, J.: Image Analysis and Mathematical Morphology, vol. 2: Theoretical Advances. Academic Press (1988)

11. Grigorescu, C., Petkov, N., Westenberg, M.: Contour detection based on nonclassical receptive field inhibition. IEEE Trans. Image Processing 12, 729-739 (2003)

12. Dubuc, B., Zucker, S.: Complexity, confusion, and perceptual grouping. Part II: Mapping complexity. J. Mathematical Imaging and Vision 15, 83-116 (2001)

13. Serra, J., Vincent, L.: An overview of morphological filtering. Circuits, Systems, and Signal Processing 11, 47-108 (1992)

14. Soille, P.: Beyond self-duality in morphological image analysis. Image and Vision Computing 23, 249-257 (2005)

15. Fukunaga, K.: Introduction to Statistical Pattern Recognition. Academic Press, Inc. (1990)

16. Salembier, P.: Comparison of some morphological segmentation algorithms based on contrast enhancement - application to automatic defect detection. In: European Signal Processing Conference, Barcelona, Spain, pp. 833-836 (September 1990)

17. Lambers, K., Zingman, I.: Towards detection of archaeological objects in highresolution remotely sensed images: the Silvretta case study. In: Proc. of Computer Appl. and Quantitative Methods in Archaeology, UK (2012) (in press)

18. Evans, A.N., Liu, X.U.: A morphological gradient approach to color edge detection. IEEE Trans. Image Processing 15, 1454-1463 (2006)

19. Hanbury, A.: The morphological top-hat operator generalised to multi-channel images. In: Proc. of the Int. Conf. on Pattern Recognition, pp. 672-675 (August 2004) 